been in place since 1999. Patients are assessed by specialist pancreatologists, surgeons, and dietitians with a consensus approach toward endoscopic intervention as a first step. This study reports the outcome of surgery in patients managed by this approach.

Methods A retrospective database searches to identify all patients having pancreatic surgery for chronic pancreatitis (CP) in the Manchester Royal Infirmary) over a 20-year period from 1999-2019. Patients were excluded from the study if final histology revealed cancer.

Results 20 patients were identified; mean (range) age in years 49 (18-80). Sixteen (80\%) were male. Alcohol was the cause in 10,3 were idiopathic with the remainder including genetic, auto-immune, and pancreas divisum. Nineteen had undergone prior endoscopic intervention. Ten patients underwent Whipple pancreatoduodenectomy, nine underwent longitudinal pancreatojejunostomy with partial head resection and there was one distal pancreatectomy. Hospital stay ranged from 6 to 32 days with a mean of 15 days. There was no operative mortality. Pancreatic exocrine insufficiency was documented in $60 \%$ before surgery and in $85 \%$ after. Diabetes was present in $55 \%$ before surgery and in $65 \%$ after.

Conclusion Multidisciplinary assessment is the key to the selection of patients to undergo surgery for chronic pancreatitis. In this selected population, surgery can be undertaken safely with low mortality.

\section{PTU-73 PRE AND POST-OP PAIN ANALYSIS IN PANCREAS SURGERY FOR CHRONIC PANCREATITIS}

Matthew Burrows*, Ala Elwasila, Ajith Siriwardena, Alistair Makin, Abubaker YM Ahmed. Manchester University Foundation Trust, Manchester, UK

\subsection{6/gutjpl-2021-BSG.275}

Introduction Chronic pancreatitis is defined as a spectrum of inflammatory disorders of the exocrine pancreas that typically result in loss of function and pain. The main indication for operating on patients with chronic pancreatitis is managing intractable pain or treating complications, however surgical intervention is usually the last resort after conservative measures have failed. Evidence from previous studies stated that early surgery has a more favorable outcome on the treatment of intractable pain compared to endoscopic management.

Aim To compare the analgesic requirements of patients who underwent surgical intervention for chronic pancreatitis preand post-operatively.

Methods A retrospective database search to identify all patients having pancreatic surgery indicated for $\mathrm{CP}$ in a tertiary center

\section{Graph Showing Change in Analgesia Requirements Post-Operatively}

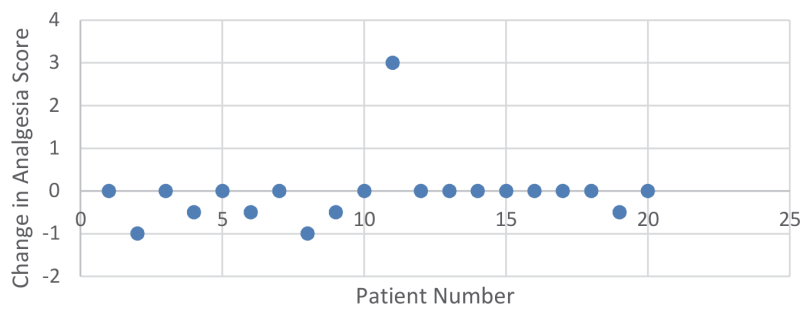

Abstract PTU-73 Figure 1
(Manchester Royal Infirmary) over a 20-year period from 1999-2019. Patients were excluded from the study if histology showing pancreatic carcinoma was identified.

Results Analgesia was scored as a 1 for simple painkillers, 2 for PRN opiates, and 3 for regular opiates with pre- and post-op cumulative scores compared. The cumulative pain score pre-operatively was 46 (5 people scoring 1,1 scoring 2, 13 scoring 3, and one patient not taking any painkillers).

Post-operatively pain score breakdown was 5 scoring 1,3 scoring 2 and 12 scoring 3, however, 4 patients that remained on regular opiates were found to be on a reduced dose compared to pre-operatively. A reduction of 0.5 was used to denote patients that remained on regular opiates but had a reduction in their dosage. Therefore the cumulative pain score was 45 .

The one patient whose analgesia requirements increased postoperatively developed a complication (pancreatic leak) post-operatively. This is believed to be the reason for the increased dependence. Excluding this patient gives a pre-operative cumulative pain score of 46 and a post-operative cumulative pain score of 42 .

Conclusion From our patient cohort, we can demonstrate a reduction in total analgesic requirements postoperatively. Whilst this remains an uncommon indication for surgical intervention it appears to hold potential benefits and merits study on a larger scale.

\section{PTU-74 WEIGHT REGRESSION ANALYSIS IN PATIENTS WHO HAVE UNDERGONE SURGERY FOR CHRONIC PANCREATITIS}

Ala Elwasila*, Matthew Burrows, Ajith Siriwardena, Alistair Makin, Abubaker YM Ahmed. Manchester University Foundation Trust, Manchester, UK

\subsection{6/gutjnl-2021-BSG.276}

Introduction Chronic pancreatitis is defined as a spectrum of inflammatory disorders of the exocrine pancreas that typically result in loss of function and pain. It often results in weight loss due to the progressive loss of endocrine and exocrine function. Surgical interventions for chronic pancreatitis are the last resort option and normally saved for patients with intractable pain however the impact of surgery on weight gain has not been previously studied.

Aim To examine the impact of surgery on weight gain in patients with chronic pancreatitis.

Methods A retrospective database search to identify all patients having pancreatic surgery indicated for $\mathrm{CP}$ in a tertiary center (Manchester Royal Infirmary) over a 20-year period from 1999-2019. Patients were excluded from the study if histology showing pancreatic carcinoma was identified. Pre-op weights were taken from nutritional screening at the time of admission for surgery. Post-op weights were taken from follow-up clinic appointments on average six months postoperatively.

Results On studying patient weights pre-and post-op it was found that $40 \%$ of patients had a steady weight gain, $25 \%$ had a weight drop, $30 \%$ had no documented weights for comparison and $5 \%$ had stable weights.

Conclusion This study has shown that over half of the cohort of patients with recorded pre and post op weights had either gained weight or kept their weight steady after surgery. This supports the hypothesis that surgical intervention in patients with chronic pancreatitis does not compromise patient weights and merits further study. 